\title{
Acute care utilization for inherited metabolic diseases among children identified through newborn screening in New York state
}

\author{
Ying Wang, PhD, $\mathrm{MPH}^{1,2}$, Marilyn Sango-Jordan, $\mathrm{MA}^{3}$ and Michele Caggana, $\mathrm{ScD}^{2,4}$
}

\begin{abstract}
Background: Currently, very few studies on long-term follow up of health outcomes and health-care utilization of children with inherited metabolic disorders identified through newborn screening are available. In this project, we analyzed acute care utilization for children with inherited metabolic disorders.

Methods: A cohort of children up to 3 years of age who were born to New York State residents in 2006-2007 with confirmed inherited metabolic disorders identified through newborn screening, was linked to hospital discharge records. For children with multiple acute care encounters (emergency department visits and/or hospital inpatient stays), rehospitalization intervals were quantified as days between a discharge date and the next encounter.
\end{abstract}

Results: Inherited metabolic disorder-related hospital care encounters were more frequent for non-Hispanic black children, Medicaid recipients, and children of younger mothers. A majority $(\sim 73 \%)$ of the children without health insurance had inherited metabolic disorder-related emergency department visits. Children with private insurance were more likely to have hospital stays. Hospital acute care utilization patterns differed with respect to inherited metabolic disorder category. Children with fatty acid oxidation disorder or organic acid disorders required the most care.

Conclusions: The information collected by the unique populationbased surveillance program about hospital care utilization for the affected children can be helpful in assessing health outcomes of the children, identifying at-risk populations, and improving access to essential medical services for children in need.

Genet Med advance online publication 13 March 2014

Key Words: acute care encounters; health-care utilization; inherited metabolic diseases; long-term follow-up; newborn screening

\section{INTRODUCTION}

Inherited metabolic disorders (IMDs) are genetic conditions that affect about 1 in 800-5,000 newborns across all disorders. ${ }^{1-5}$ IMDs may lead to severe infant morbidity or death if undetected and untreated within days after birth. Expanded newborn screening (NBS) has resulted in early identification of newborns with IMDs. ${ }^{6,7}$

Long-term follow-up programs are necessary for children with inherited conditions identified through NBS programs to ensure timely and adequate medical care and treatment. ${ }^{8}$ Currently, very few studies on long-term follow-up of health outcomes and health-care utilization of children with NBS disorders are available. Studies have shown that information about long-term outcomes of affected children is critical and valuable for evaluating the effectiveness of medical treatments and services, monitoring health-care utilization of affected individuals, and estimating the prevalence or incidence of the disease. ${ }^{9-15}$ This study analyzed acute care utilization patterns of children with IMDs during their first 3 years of life using available administrative data sources.

\section{MATERIALS AND METHODS}

\section{Data sources}

NBS program. The NBS Program, under the direction of the Wadsworth Center's Division of Genetics of the New York State Department of Health (NYSDOH), performs screening tests for more than 40 disorders among 250,000 newborns annually. Each year, about 75-100 infants with IMDs are identified in NYS. ${ }^{16}$ Information collected by the NBS Program includes child and maternal demographics, screening and confirmatory tests, and health-care provider's contact information.

Hospital discharges. Hospital inpatient and emergency department (ED) discharge data were obtained from the NYSDOH's Statewide Planning and Research Cooperative System (SPARCS). The patient-level discharge records contain patient information such as date of birth, date of discharges, medical record numbers, and diagnoses.

The NYSDOH's institutional review board approved the study protocol and the project staff signed data-use agreements prior to handling the data sets.

${ }^{1}$ Division of Data Management and Research, Office of Primary Care and Health Management System, New York State Department of Health, Albany, New York, USA; ${ }^{2}$ School of Public Health, University at Albany, State University of New York, Albany, New York, USA; ${ }^{3}$ Bureau of Healthcare-Associated Infections, New York State Department of Health, Albany, New York, USA; ${ }^{4}$ Newborn Screening Program, Wadsworth Center, New York State Department of Health, Albany, New York, USA. Correspondence: Ying Wang (wxy01@ health.state.ny.us) 


\section{Study cohort}

Children with confirmed IMDs born in 2006-2007 to state residents were identified from the NBS database for inclusion in the study. Children who died during the first 3 years after birth were excluded. Birth and maternal risk factors were obtained by matching the study cohort to the birth records maintained by the NYSDOH. The study cohort was also matched with SPARCS hospital discharges to obtain information about health-care service utilization for children with IMDs for up to 3 years after birth.

\section{Inherited metabolic disorders}

In this study, we grouped the IMDs into four categories: amino acid, fatty acid oxidation, organic acid, and urea cycle disorders. The individual conditions in each category are shown in Supplementary Appendix 1. Owing to small numbers of individual IMDs, we used the four IMD categories in the analysis.

\section{Data linkage}

As described elsewhere, ${ }^{17}$ owing to lack of common identifiers between data sources, we used multiple identifying variables including name, date of birth, and address to conduct data matching deterministically. To identify correct matches, we manually examined all potential matches.

\section{Hospital care encounters}

Acute care encounters were defined as treat-and-release ED visits or hospital inpatient stays, including admissions through the ED. ED visits and inpatient information were obtained from the SPARCS hospital discharge files for children who were matched to SPARCS data. Children who were not matched to SPARCS were defined as having no acute health-care encounters. The acute care encounters were categorized into two groups: IMDrelated encounters and non-IMD related encounters. The narrative and International Classification of Diseases, Ninth Revision codes for these IMD-related encounters/conditions are listed in Supplementary Appendix 2.

\section{Data analysis}

We conducted descriptive analysis using the statistical analysis system (SAS) software (SAS Institute, Cary, NC). The standard error of the percentage was calculated using a binomial distribution at a $95 \%$ confidence level.

\section{RESULTS}

A total of 180 newborns with confirmed IMDs were ascertained through the NYS NBS Program among nearly half a million live births to NYS residents during the years 2006-2007. All 180 newborns were matched to their birth certificates; 148 (82\%) were matched to SPARCS hospital discharge files, and $32(18 \%)$ were not. A summary of the study cohort is shown in Table 1. The study cohort was composed of $54.4 \%$ males, and $52.2 \%$ non-Hispanic white, $17.8 \%$ non-Hispanic black, and $12.8 \%$ Hispanic children. Nearly half $(45.0 \%)$ of the children had an organic acid disorder.

Table 2 presents acute care encounter status of the study cohort by patient characteristics during the first 3 years of life. Overall, 73 (40.6\%) of the children had IMD-related acute care encounters (including children who had both IMD-related and non-IMD-related encounters), 75 (41.7\%) had only nonIMD-related acute care encounters, and 32 (17.8\%) had no acute care encounters during their first 3 years of life. We categorized these 32 children as having "no encounters" because they were not matched to the hospital discharge files and thus had no payment (health insurance) information. Children born to teen mothers were more likely (52.4\%) to have IMD-related encounters compared with children of older mothers. However, this observed difference was not statistically significant. A

Table 1 Summary of the study cohort by patient characteristics (New York State births 2006-2007 with confirmed IMDs, $n=180)$

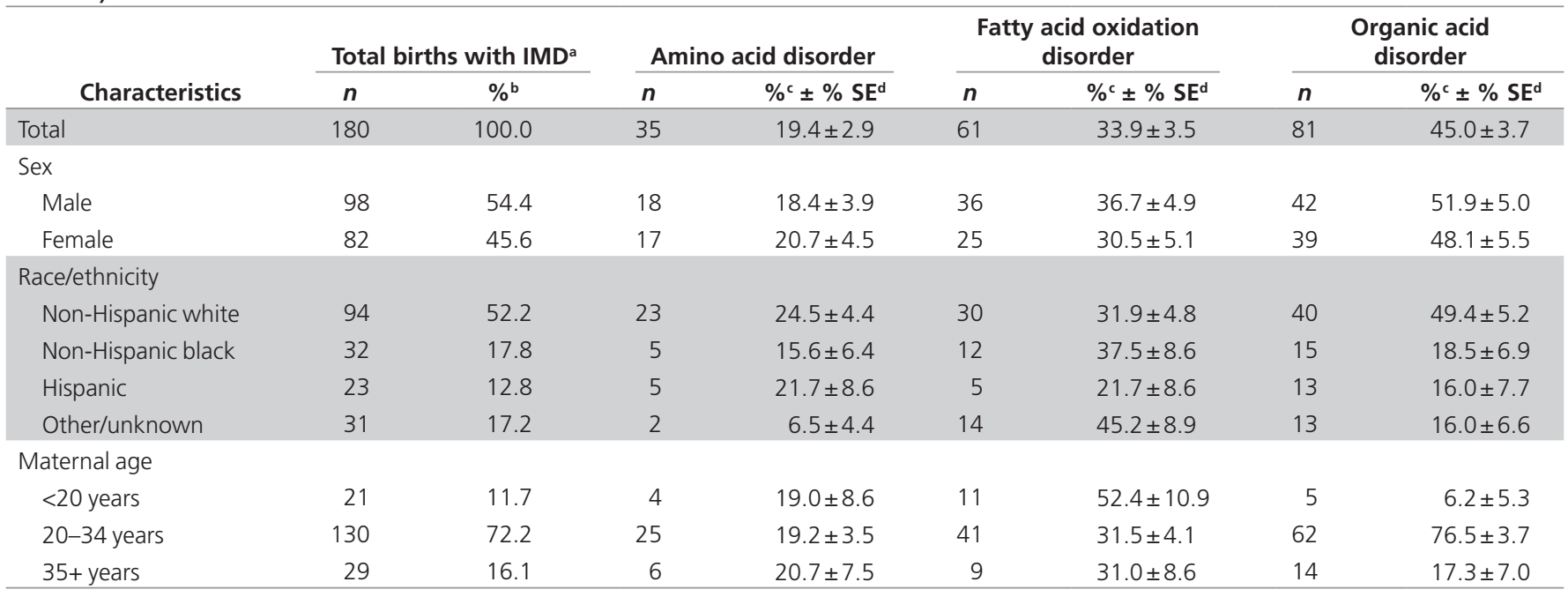

IMD, inherited metabolic disorder.

ancludes the three urea cycle disorder cases. ${ }^{b}$ Column percentage. ${ }^{\text {CRow }}$ percentage. ${ }^{\mathrm{d} S t a n d a r d}$ error. 
Table 2 Acute care encounters (emergency department visits or hospital inpatient stays) status during the first 3 years of life by patient characteristics (New York State births 2006-2007 with confirmed inherited metabolic disorders, $n=180$ )

\begin{tabular}{|c|c|c|c|c|c|c|c|}
\hline Characteristics & $\begin{array}{c}\text { Total } \\
\text { patients }\end{array}$ & \multicolumn{2}{|c|}{ IMD-related encounters ${ }^{a}$} & \multicolumn{2}{|c|}{$\begin{array}{l}\text { Non-IMD-related } \\
\text { encounters }\end{array}$} & \multicolumn{2}{|c|}{ No encounter ${ }^{b}$} \\
\hline Total & 180 & 73 & $40.6 \pm 3.7$ & 75 & $41.7 \pm 3.7$ & 32 & $17.8 \pm 2.8$ \\
\hline Non-Hispanic white & 94 & 37 & $39.4 \pm 5.0$ & 39 & $41.5 \pm 5.1$ & 18 & $19.1 \pm 4.1$ \\
\hline Non-Hispanic black & 32 & 15 & $46.9 \pm 8.8$ & 13 & $40.6 \pm 8.7$ & 4 & $12.5 \pm 5.8$ \\
\hline Hispanic & 23 & 9 & $39.1 \pm 10.2$ & 10 & $43.5 \pm 10.3$ & 4 & $17.4 \pm 7.9$ \\
\hline \multicolumn{8}{|l|}{ Maternal age } \\
\hline$<20$ years & 21 & 11 & $52.4 \pm 10.9$ & 9 & $42.9 \pm 10.8$ & 1 & $4.8 \pm 4.6$ \\
\hline 20-34 years & 130 & 52 & $40.0 \pm 4.3$ & 53 & $40.8 \pm 4.3$ & 25 & $19.2 \pm 3.5$ \\
\hline $35+$ years & 29 & 10 & $34.5 \pm 8.8$ & 13 & $44.8 \pm 9.2$ & 6 & $20.7 \pm 7.5$ \\
\hline \multicolumn{8}{|l|}{ Payment type } \\
\hline Private insurance & 62 & 25 & $40.3 \pm 6.2$ & 37 & $59.7 \pm 6.2$ & - & - \\
\hline \multicolumn{8}{|l|}{ IMD category ${ }^{d}$} \\
\hline Amino acid disorders & 35 & 13 & $37.1 \pm 8.2$ & 12 & $34.3 \pm 8.0$ & 10 & $28.6 \pm 7.6$ \\
\hline Fatty acid oxidation disorders & 61 & 27 & $44.3 \pm 6.4$ & 27 & $44.3 \pm 6.4$ & 7 & $11.5 \pm 4.1$ \\
\hline Organic acid disorders & 81 & 32 & $39.5 \pm 5.4$ & 35 & $43.2 \pm 5.5$ & 14 & $17.3 \pm 4.2$ \\
\hline
\end{tabular}

IMD, inherited metabolic disorder.

ancludes cases with both IMD-related and non-IMD-related encounters. ${ }^{b} 32$ Cases were not found in hospital discharge data (inpatient and outpatient files); thus, no payment/insurance information was found. 'Standard error. ${ }^{\text {T}}$ Three cases with urea cycle disorders were excluded because of small numbers

statistically significant higher percentage of children with Medicaid had IMD-related encounters (52.1\%) compared with children with private insurance (40.3\%). No significant difference regarding IMD-related or non-IMD-related encounters was observed for race/ethnicity or IMD categories.

Table 3 presents the acute care encounter type (ED visit or hospitalization) by patient characteristics for the 73 children who had IMD-related encounters during their first 3 years of life. Among these children, 30 (41.1\%) had ED visits and 43 (58.9\%) had hospital inpatient stays, including those admitted through the ED and those who had both ED visits and inpatient stays. A statistically significant higher percentage of Hispanic children had ED visits (55.6\%) compared with non-Hispanic black children (26.7\%). Children with private health insurance or Medicaid had a statistically significant higher hospitalization rate $(68.0 \%$ for private insurance; $62.2 \%$ for Medicaid) than children without health insurance (including unknowns; 27.3\%). Children without health insurance (including unknowns) had a statistically significant higher rate of ED visits (72.7\%) compared with children with health insurance $(32.0 \%$ for private insurance and $37.8 \%$ for Medicaid). No statistically significant difference in the encounter type was found among the three IMD categories.

Table 4 shows IMD-related acute care encounter frequency, repeat encounters within 30 days, and inpatient length of stay (LOS) by IMD category during the first 3 years of life. Among the 72 IMD children, 47 (65.3\%) had only one IMDrelated encounter and $25(34.7 \%)$ had two or more encounters.
Children with fatty acid oxidation or organic acid disorders were more likely to have two or more encounters compared with children with amino acid disorders, although the difference was not statistically significant. Among the 25 children with two or more encounters, 7 (28.0\%) had repeated visits (ED or hospital inpatient stay) within 30 days; 3 had fatty acid oxidation disorders and 4 had organic acid disorders.

For the 42 children who had hospital inpatient stays, the average and median LOSs were 23 and 4 days, respectively, with the longest LOS among children with fatty acid oxidation disorders (mean: 46 days; median: 10 days) and the shortest LOS among children with organic acid disorders (8 days). Two-thirds (28/42) of the infants had 1-10 days of stay. About $38 \%$ (16 of 42 infants; $37 \%$ for amino acid disorders, $40 \%$ for fatty acid disorders, and $37 \%$ for organic acid disorders) of the study cohort had IMD-related inpatient stays right after birth, with an average LOS of 25 days, ranging from 2 to 185 days (a child with a fatty acid oxidation disorder; data not shown). Based on the reported International Classification of Diseases, Ninth Revision codes, congenital respiratory disorders were the most common comorbidities among infants with amino acid disorders; perinatal jaundice was the most common comorbidity among infants with fatty acid oxidation or organic acid disorders.

\section{DISCUSSION}

Administrative databases have been used by researchers to monitor health care-service access and utilization for targeted, at-risk 
Table 3 Inherited metabolic disorder-related acute care encounters (emergency department visits or hospital inpatient stays) during the first 3 years of life by patient characteristics and type of health care (New York State births 2006-2007 with IMDs, $n=73$ )

\begin{tabular}{|c|c|c|c|c|c|c|}
\hline \multirow[b]{2}{*}{ Characteristics } & \multicolumn{2}{|c|}{ Total patients } & \multicolumn{2}{|c|}{ Emergency department visits ${ }^{a}$} & \multicolumn{2}{|c|}{ Inpatient stays ${ }^{b}$} \\
\hline & $n$ & $\%^{c}$ & $n$ & $\%^{\mathrm{d}} \pm \% S \mathrm{SE}^{\mathrm{e}}$ & $n$ & $\%^{d} \pm \% S E^{e}$ \\
\hline Total & 73 & 100.0 & 30 & $41.1 \pm 5.8$ & 43 & $58.9 \pm 5.8$ \\
\hline \multicolumn{7}{|l|}{ Race/ethnicity } \\
\hline Non-Hispanic white & 37 & 50.7 & 16 & $43.2 \pm 8.1$ & 21 & $56.8 \pm 8.1$ \\
\hline Non-Hispanic black & 15 & 20.5 & 4 & $26.7 \pm 11.4$ & 11 & $73.3 \pm 11.4$ \\
\hline \multicolumn{7}{|l|}{ Maternal age } \\
\hline$<20$ years & 11 & 15.1 & 4 & $36.4 \pm 14.5$ & 7 & $63.6 \pm 14.5$ \\
\hline 20-34 years & 52 & 71.2 & 22 & $42.3 \pm 6.9$ & 30 & $57.7 \pm 6.9$ \\
\hline $35+$ years & 10 & 13.7 & 4 & $40.0 \pm 15.5$ & 6 & $60.0 \pm 15.5$ \\
\hline Self-pay/unknown & 11 & 15.1 & 8 & $72.7 \pm 13.4$ & 3 & $27.3 \pm 13.4$ \\
\hline \multicolumn{7}{|l|}{ IMD category } \\
\hline Amino acid disorders & 13 & 17.8 & 5 & $38.5 \pm 13.5$ & 8 & $61.5 \pm 13.5$ \\
\hline Fatty acid oxidation disorders & 27 & 37.0 & 12 & $44.4 \pm 9.6$ & 15 & $55.6 \pm 9.6$ \\
\hline Organic acid disorders & 32 & 43.8 & 13 & $40.6 \pm 8.7$ & 19 & $59.4 \pm 8.7$ \\
\hline
\end{tabular}

IMD, inherited metabolic disorder.

${ }^{a}$ Emergency department (ED) visits that did not result in a hospital inpatient admission. ${ }^{b}$ Inpatient stays (including admission through the ED) and those with both ED visits and inpatient stays. 'Column percentage. ${ }^{d}$ Row percentage. ${ }^{e S t a n d a r d ~ e r r o r . ~}{ }^{\mathrm{f}}$ Excludes urea cycle disorders due to small numbers.

Table 4 The number of acute care encounters (emergency department visits or hospital inpatient stays) and inpatient length of stay during the first 3 years of life by inherited metabolic disorder category (New York State births 2006-2007 with confirmed IMDs $n=72$, excluding the case with urea cycle disorder)

\begin{tabular}{|c|c|c|c|c|c|c|c|c|c|c|c|c|c|}
\hline \multirow[b]{3}{*}{ IMD category } & \multirow{3}{*}{$\begin{array}{c}\text { Total } \\
\text { patients }\end{array}$} & \multirow{2}{*}{\multicolumn{2}{|c|}{$\begin{array}{l}\text { Had one } \\
\text { encounter }\end{array}$}} & \multirow{2}{*}{\multicolumn{2}{|c|}{$\begin{array}{c}\text { Had two } \\
\text { or more } \\
\text { encounters }\end{array}$}} & \multirow{2}{*}{\multicolumn{2}{|c|}{$\begin{array}{l}\text { Had two or more } \\
\text { encounters }(n=25) \text { : } \\
\text { revisits within } 30 \text { days }\end{array}$}} & \multicolumn{6}{|c|}{ LOS for children with inpatient stays $(n=42)$} \\
\hline & & & & & & & & \multicolumn{2}{|c|}{ LOS, days } & \multicolumn{4}{|c|}{ Children with LOS } \\
\hline & & $n$ & $\% \pm \% \mathrm{SE}$ & $n$ & $\% \pm \% \mathrm{SE}$ & $n$ & $\% \pm \% \mathrm{SE}$ & Mean & Median & $1-10$ & $11-30$ & $>30$ & Total \\
\hline $\begin{array}{l}\text { Amino acid } \\
\text { disorders }\end{array}$ & 13 & 10 & $76.9 \pm 11.7$ & 3 & $23.1 \pm 11.7$ & 0 & $0.0 \pm 0.0$ & 18 & 3 & 5 & 1 & 2 & 8 \\
\hline $\begin{array}{l}\text { Fatty acid } \\
\text { oxidation disorders }\end{array}$ & 27 & 17 & $63.0 \pm 9.3$ & 10 & $37.0 \pm 9.3$ & 3 & $30.0 \pm 14.5$ & 46 & 10 & 8 & 2 & 5 & 15 \\
\hline Total & 72 & 47 & $65.3 \pm 5.6$ & 25 & $34.7 \pm 5.6$ & 7 & $28.0 \pm 9.0$ & 23 & 4 & 28 & 5 & 9 & 42 \\
\hline
\end{tabular}

IMD, inherited metabolic disorder; LOS, length of stay; SE, standard error.

populations. ${ }^{18-21}$ Using the NYSDOH SPARCS hospital discharge files, we were able to collect information on health-care utilization of IMD children during their first 3 years after birth.

We found that about $41 \%$ of the children with IMDs had IMD-related acute care encounters during their first 3 years of life, including ED visits and/or inpatient hospitalization. The hospital utilization rates of the children with IMDs were affected by demographic characteristics. IMD-related acute care hospital utilization was more frequent for Medicaid recipients and children of teen mothers. These findings are consistent with those reported previously. A study on health-care utilization and expenditures for privately and publicly insured children with sickle-cell disease showed that the percentage of Medicaid-enrolled children with sickle-cell disease having inpatient admissions was higher than that for privately insured children. ${ }^{22}$ 
The current study found that a majority $(\sim 73 \%)$ of the children who had an acute care encounter without documented health insurance had IMD-related ED visits and that a higher percentage of IMD children with private insurance had hospital inpatient stays. Our finding that Hispanic children were more likely to have IMD-related ED visits is consistent with the result from a study on disparities in health care-service utilization in California among Latino children with asthma, ${ }^{23}$ which reported that Latino children suffering from asthma were $49 \%$ more likely to have visited an ED compared with non-Latino children with asthma. A survey study reported that maternal/ family characteristics were the major contributing factors to racial/ethnic disparities in health-care utilization. ${ }^{24}$

In the current study, about $18 \%$ of the children with IMDs were not found in SPARCS hospital discharge files (including both inpatient and outpatient records). It should be noted that some of these children may not have been found in the discharge files because their NBS and vital statistics records were discrepant with the discharge data. The lack of patient name in SPARCS data set made it difficult to achieve a high percentage of matching. By using multiple matching criteria including patient medical record numbers and other available demographic variables, we were able to match $82 \%$ of the study cohort. We could have missed some true matches, but it is difficult to estimate this figure.

Another limitation of this study was that we were not able to present the analysis results for individual IMD conditions due to the rareness of the conditions. Even though we used categorized data, some of the analysis results have large standard errors due to small numbers in the stratified groups, leading to unstable results. Thus, the findings from the current study should be used and generalized with caution. The Centers for Disease Control and Prevention has funded four states (including New York) for a pilot project on developing a statewide, population-based surveillance system for children with confirmed metabolic disorders (19 selected conditions). ${ }^{17,25}$ Data on health outcomes and health care-service utilization of the affected children have been collected. Analysis will be conducted using pooled data from the four state programs to increase the statistical power for some rare conditions.

In conclusion, using the administrative data and the SPARCS hospital discharge files, we followed children with IMDs up to 3 years after birth and collected information about health-care utilization. This systematic follow-up and data-collection approach through data linkage with available administrative data has proven to be efficient and cost-effective. The information collected by the unique population-based surveillance program about hospital care utilization for the affected children can be helpful in assessing health outcomes of the children and identifying at-risk populations and essential medical services for children in need.

\section{SUPPLEMENTARY MATERIAL}

Supplementary material is linked to the online version of the paper at http://www.nature.com/gim

\section{ACKNOWLEDGMENT}

This project was funded by US Centers for Disease Control and Prevention grant 1U50DD000474-01.

\section{DISCLOSURE}

The authors declare no conflict of interest.

\section{REFERENCES}

1. Sanderson S, Green A, Preece MA, Burton $H$. The incidence of inherited metabolic disorders in the West Midlands, UK. Arch Dis Child 2006; 91:896-899.

2. Applegarth DA, Toone JR, Lowry RB. Incidence of inborn errors of metabolism in British Columbia, 1969-1996. Pediatrics 2000;105:e10.

3. Martins AM. Inborn errors of metabolism: a clinical overview. Sao Paulo Med J 1999;117:251-265.

4. Seymour CA, Thomason MJ, Chalmers RA, et al. Newborn screening for inborn errors of metabolism: a systematic review. Health Technol Assess 1997; 1:i-iv, 1

5. Dionisi-Vici C, Rizzo C, Burlina AB, et al. Inborn errors of metabolism in the Italian pediatric population: a national retrospective survey. J Pediatr 2002;140:321-327.

6. Schulze A, Lindner M, Kohlmüller D, Olgemöller K, Mayatepek E, Hoffmann GF. Expanded newborn screening for inborn errors of metabolism by electrospray ionization-tandem mass spectrometry: results, outcome, and implications. Pediatrics 2003;111(6 Pt 1):1399-1406.

7. Sweetman L, Millington DS, Therrell BL, et al. Naming and counting disorders (conditions) included in newborn screening panels. Pediatrics 2006;117/5 Pt 2):S308-S314.

8. Kemper AR, Boyle CA, Aceves J, et al. Long-term follow-up after diagnosis resulting from newborn screening: statement of the US Secretary of Health and Human Services' Advisory Committee on Heritable Disorders and Genetic Diseases in Newborns and Children. Genet Med 2008;10:259-261.

9. Centers for Disease Control and Prevention (CDC). Evaluating newborn screening program data systems-Georgia, 1998. MMWR Morb Mortal Wkly Rep 1999;48:1101-1104.

10. Dawson AL, Cassell CH, Riehle-Colarusso T, et al. Factors associated with late detection of critical congenital heart disease in newborns. Pediatrics 2013;132:e604-e611.

11. Van Naarden Braun K, Yeargin-Allsopp M, Schendel D, Fernhoff P. Long-term developmental outcomes of children identified through a newborn screening program with a metabolic or endocrine disorder: a population-based approach. J Pediatr 2003;143:236-242.

12. Powell K, Van Naarden Braun K, Singh R, Shapira SK, Olney RS, YearginAllsopp M. Prevalence of developmental disabilities and receipt of special education services among children with an inborn error of metabolism. J Pediatr 2010;156:420-426.

13. Aoki K, Ohwada M, Kitagawa T. Long-term follow-up study of patients with phenylketonuria detected by the newborn screening programme in Japan. J Inherit Metab Dis 2007;30:608.

14. Sahai I, Eaton RB, Hale JE, Mulcahy EA, Comeau AM. Long-term followup to ensure quality care of individuals diagnosed with newborn screening conditions: early experience in New England. Genet Med 2010;12(12 suppl):S220-\$227.

15. Couce ML, Castiñeiras DE, Bóveda MD, et al. Evaluation and long-term follow-up of infants with inborn errors of metabolism identified in an expanded screening programme. Mol Genet Metab 2011;104:470475.

16. Newborn Screening Program, New York State Department of Health. 2010 Annual Reports, online publications. http://www.wadsworth.org/newborn/ annualrept/annsum.htm. Accessed 16 April 2011

17. Wang Y, Caggana M, Sango-Jordan M, Sun M, Druschel CM. Long-term follow-up of children with confirmed newborn screening disorders using record linkage. Genet Med 2011;13:881-886.

18. Collins AJ, Chen SC, Gilbertson DT, Foley RN. CKD surveillance using administrative data: impact on the health care system. Am J Kidney Dis 2009;53(3 suppl 3):S27-S36

19. Evans E, Grella CE, Murphy DA, Hser YI. Using administrative data for longitudinal substance abuse research. J Behav Health Serv Res 2010;37:252271. 


\section{ORIGINAL RESEARCH ARTICLE}

20. Grigorescu V, Kleyn MJ, Korzeniewski SJ, Young WI, Whitten-Shurney W. Newborn screening follow-up within the lifespan context: Michigan's experience. Am J Prev Med 2010;38(4 suppl):S522-S527.

21. Grosse SD, Boulet SL, Amendah DD, Oyeku SO. Administrative data sets and health services research on hemoglobinopathies: a review of the literature. Am J Prev Med 2010;38(4 suppl):S557-S567.

22. Mvundura M, Amendah D, Kavanagh PL, Sprinz PG, Grosse SD. Health care utilization and expenditures for privately and publicly insured children with sickle cell disease in the United States. Pediatr Blood Cancer 2009;53:642-646.
WANG et al | Hospital care utilization for children with inherited metabolic diseases

23. Chang J, Patel I, Liu ST, et al. Disparities in health care utilization among Latino children suffering from asthma in California. Pediatr Health Med Ther $2011 ; 2: 1-8$.

24. Flores G, Olson L, Tomany-Korman SC. Racial and ethnic disparities in early childhood health and health care. Pediatrics 2005;115:e183-e193.

25. Hinton CF, Mai CT, Nabukera SK, et al. Developing a public health-tracking system for follow-up of newborn screening metabolic conditions: a four-state pilot project structure and initial findings. Genet Med 2013; e-pub ahead of print 5 December 2013 